Check for updates

Cite this: Phys. Chem. Chem. Phys.,

2021, 23, 3327

Received 22nd September 2020,

Accepted 20th January 2021

DOI: $10.1039 / \mathrm{d} 0 \mathrm{cp} 05007 \mathrm{e}$

rsc.li/pccp

\section{The electronic structure of carbones revealed: insights from valence bond theory $\dagger$}

\author{
Remco W. A. Havenith, (D) *abc Ana V. Cunha, d Johannes E. M. N. Klein, (D) ${ }^{a}$ \\ Francesca Perolari ${ }^{b}$ and Xintao Feng ${ }^{a}$
}

In this contribution, we studied the $\mathrm{OC}-\mathrm{C}$ bond in carbon suboxide and related allene compounds using the valence bond method. The nature of this bond has been the subject of debate, whether it is a regular, electron sharing bond or a dative bond. We compared the nature of this bond in carbon suboxide with the gold-CO bond in $\mathrm{Au}(\mathrm{CO})_{2}{ }^{+}$, which is a typical dative bond, and we studied its charge-shift bond character. We found that the $\mathrm{C}-\mathrm{CO}$ bond in carbon suboxide is unique in the sense that it cannot be assigned as either a dative or electron sharing bond, but it is an admixture of electron sharing and dative components, together with a high contribution of ionic character. These findings provide a clear basis for distinguishing the commonly found dative bonds between ligands and transition metals and the present case of what may be described as coordinative bonding to carbon.

\section{Introduction}

The nature of the chemical bond has been occupying chemists from the dawn of chemistry. The notions of electron sharing to form a covalent bond, and the octet rule have been and still are very successful concepts to explain structure and reactivity of molecules. However, even small molecules, that, at first sight, look straightforward and follow the rules, show a richer chemistry than expected based on the simplest Lewis structures.

Frenking and coworkers studied carbon suboxide (1), related allenenic compounds, and carbodiphosphoranes (Scheme 1). ${ }^{1-6}$ Using various computational techniques, they studied the nature of the OC-C bond. ${ }^{3,7}$ A main question in this research was to explain the bonding in $\mathbf{1}$, as $\mathbf{1}$ shows a very shallow $\mathrm{C}-\mathrm{C}-\mathrm{C}$ bending potential ${ }^{8-10}$ and it is argued that it is slightly bent $\left(156^{\circ}\right)$ in the gas phase. ${ }^{11}$ In the solid state, it is on average linear, but large vibrational ellipsoids of the oxygen and central carbon

\footnotetext{
${ }^{a}$ Stratingh Institute for Chemistry, University of Groningen, Nijenborgh 4, 9747 AG Groningen, The Netherlands. E-mail: r.w.a.havenith@rug.nl

${ }^{b}$ Zernike Institute for Advanced Materials, University of Groningen, Nijenborgh 4, 9747 AG Groningen, The Netherlands

${ }^{c}$ Ghent Quantum Chemistry Group, Department of Inorganic and Physical Chemistry, Ghent University, Krijgslaan 281 (S3), B-9000 Gent, Belgium

${ }^{d}$ Scientific Computing Group, Oak Ridge National Laboratory, Oak Ridge, TN 37831-6373, USA

$\dagger$ Electronic supplementary information (ESI) available: Tables S1-S5, containing the Cartesian coordinates of $\mathbf{1 - 5}$, a justification for the use of fragment restricted orbitals (including Table S6 (GN/CC weights of the structures) and Fig. S1 (VBSCF orbitals for 1 without any restrictions), Table S7 with all total energies (VB and CASSCF), Table S8 with all EDA components, Table S9, the combined CC weights for 1-5, and Table S10, containing the orbital overlaps. See DOI: 10.1039/ d0cp05007e
}

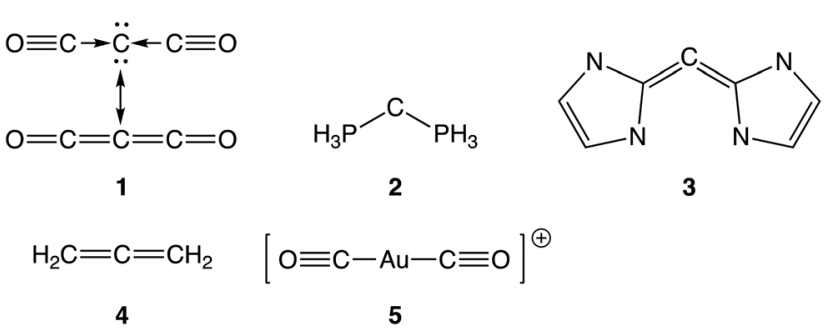

Scheme 1 Structures of the molecule studied in this contribution.

atoms are visible. ${ }^{12}$ This very flexible $\mathrm{C}-\mathrm{C}-\mathrm{C}$ bending mode and the non-linear structure was also confirmed with coupled cluster calculations. ${ }^{13}$ Frenking and coworkers performed extensive energy decomposition analyses (EDA), ${ }^{14}$ using different fragments that can be envisioned to compose the bond, such as singlet/triplet CO that forms a bond with the ${ }^{1} \mathrm{D} /{ }^{5} \mathrm{~S}$ of carbon, respectively. ${ }^{3}$ They not only looked at the orbital interaction terms, but also at the excitation energies for the fragments to access the so-called 'reactive state' from their ground state i.e., the electronic state that the fragments should have to form the bonds. This extensive analysis showed that the CO-C $\sigma$-bond could be best described as a dative bond from $\mathrm{CO}$ to the central $\mathrm{C}$ atom. Thus, they proposed that the $\mathrm{C}$ atom in $\mathbf{1}$ is in an oxidation state of 0 , and that it formally has two lone pairs and accepts two dative bonds from the two CO moieties, and termed it a carbone. ${ }^{15}$ This conclusion was not only based on EDA, but was further substantiated with NBO analyses, proton affinities and reactivity studies, confirming the formal oxidation state of 0 , that would be derived from the existence of dative bonds towards carbon. ${ }^{7}$ 
Not only 1 exhibits a central carbon atom that can accept dative bonding: the allenes, such as $\mathbf{3}$ and $\mathbf{4}$, can also be viewed as a central carbon(0) atom that accepts two dative bonds from a carbene $\mathrm{CL}_{2}\left(\left(\mathrm{CL}_{2}\right) \mathrm{C}\left(\mathrm{CL}_{2}\right)\right)$. Different allenes (with different ligands L) have been the subject of a study towards the nature of the central $\mathrm{C}=\mathrm{C}$ bond, and similar conclusions were drawn: these allenenic structures have carbone character. ${ }^{2,3,5,7,16,17}$ Carbodiphosphorane 2 was also computationally intensively studied, ${ }^{1,18}$ and a more conclusive picture emerged: carbon is able to accept dative bonds, while being in a formal oxidation state of 0 . It was found that if the singlet/triplet splitting of the ligand is high and the singlet is favored, the carbone character is higher. ${ }^{18}$ This view of dative bonding to carbon (for an example of using the term coordination chemistry in this context see ref. 19) was able to explain the non-linear geometry of the aforementioned allenes. The dative bonding and the existence of the two lone-pairs on the central carbon atom would lead to bent geometries, whereas the traditional viewpoint of two double, electron sharing, bonds would suggest a linear geometry.

However, this view was disputed in the literature. ${ }^{20-22}$ Discussions erupted whether it was really necessary to introduce more 'arrow drawing' in chemistry, and it was questioned if terming these bonds to carbon as dative added a valuable perspective. It was argued that the traditional view with ionic resonance structures could equally well explain the bent geometry of $\mathbf{1}$ and related compounds and their observed chemistry. These two viewpoints remain to co-exist and have not been unified into one "final" concept.

In an attempt to provide a unified view, valence bond (VB) theory $^{23}$ is applied to interpret the wavefunctions of these molecules, where we provide the basis for differentiating dative bonding in carbones when compared to classical transition metal complexes. The heavier homologues of $\mathbf{1}$ have been previously studied using VB theory, ${ }^{24}$ but 1 itself was left out. VB can offer a view to chemical bonding much closer to the commonly used Lewis structures than traditional, orthogonal, molecular orbital theories. In VB theory, a bond is represented by the spin-coupling of two electrons in two, mutually nonorthogonal, singly occupied orbitals. These singly occupied orbitals are atomic in nature. Using spin-coupled bonds, a wavefunction for a VB structure can be built that resembles one of the possible Lewis structures. The final VB wavefunction is written as a linear superposition of the possible VB structures. Each VB structure is then a linear combination of Slater determinants with fixed spin-coupling coefficients, resembling one particular binding motif, or Lewis structure. The expansion coefficients for the VB structures in the final VB wavefunction are variationally optimized, and the orbitals that comprise the determinants in each structure are simultaneously optimized. During the orbital optimization, the orbitals may be kept localized on atoms or particular molecular fragments, or may be allowed to delocalize over the entire molecule. ${ }^{25-27}$ It is also possible to use different orbitals for different VB structures (breathing orbital method ${ }^{28-31}$ ). The VB treatment offers a wavefunction that can directly be interpreted from a Lewis

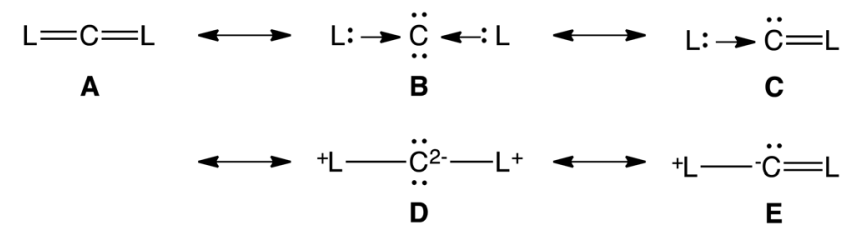

Scheme 2 The five types of valence bond structures considered in this work.

structure perspective, and provides insight into the importance of a particular structure/representation for the total description of the molecule. It also provides the set of optimized orbitals that form the chemical bonds in the molecule, which will aid in the interpretation of the bonding motifs in a molecule, by aiding in the interpretation of the VB structure. Furthermore, it provides energies of the individual structures, and an energy for the final wavefunction, thus a stabilization energy due to resonance between VB structures is unambiguously defined. ${ }^{32,33}$ Different flavours of VB theory exists, e.g., the spin-coupled VB method mi-37 $^{34}$ and the Generalized VB method, ${ }^{38-40}$ but we opt here for the valence bond self-consistent field (VBSCF) method. ${ }^{25,26}$

In this contribution, we performed VBSCF calculations and an interpretation of the VBSCF wavefunctions in terms of different bonding motifs for selected carbones, together with reference molecules containing traditional electron sharing and dative bonding (Scheme 1). The molecules 1-3 are reported to have high degrees of carbone character, whereas 4 is considered as a traditional allene with two traditional electron sharing bonds. The gold $(\mathrm{I})(\mathrm{CO})_{2}{ }^{+}$complex 5 is a typical complex with two dative bonds from $\mathrm{CO}$ to $\mathrm{Au}$ as commonly found in coordination chemistry; there is little $\pi$-backbonding from the gold towards $\mathrm{CO}$, making the $\mathrm{Au}-\mathrm{CO}$ bonds clean dative bonds. ${ }^{41-43}$

In our study, we considered the VB structures as depicted in Scheme 2. Structure A represents the traditional electron sharing bond, structure $\mathbf{B}$ is the carbone structure with two dative bonds, and structure $\mathbf{C}$ is a carbene-like model, with one dative and one covalent double bond. The structures $\mathbf{D}$ and $\mathbf{E}$ represent the ionic structures, which are invoked to explain the bonding in 2. We have chosen to keep the orbitals in the valence bond structures localized on the ligands (L) and on the central carbon atom. The electron pairs depicted in Scheme 2 are described by spin-coupled bonds, while the remaining bonds of the ligands are described by doubly occupied, orthogonal bonds.

\section{Computational details}

Geometries were optimized with ADF, ${ }^{44-46}$ using the TZ2P basis set and BP86 functional (no symmetry constraints). Energy decomposition analyses were also performed with ADF. ${ }^{14}$ Hessian calculations were performed to confirm that all optimized structures are local minima at this level of theory. For 5, scalar relativistic effects were included via the ZORA method. ${ }^{47-49}$

For 1, CCSD/cc-pVTZ geometry optimization and CCSD angle scan were done using Gaussian $16,{ }^{50}$ using the cc-pVTZ basis set. $^{51}$ 
Valence bond SCF calculations ${ }^{25,26}$ were performed with TURTLE, ${ }^{52,53}$ as implemented in GAMESS-UK. ${ }^{54}$ For the Valence Bond calculations, the cc-pVDZ (gold: cc-pVDZ-PP) basis set ${ }^{51,55-57}$ was used. Basis sets were taken from the BasisSet Exchange Library. ${ }^{58-60}$ The molecules were divided in three fragments, viz. the central carbon atom, and each of the two ligands. The orbitals were kept localized on each fragment (for a justification of this approach, see the ESI $\dagger$ ). Two sets of valence bond calculations were performed: (1) Only the neutral valence bond structures depicted in Scheme 2 were used: this resulted in 7 structures for $\mathbf{1}(2 \times \mathbf{A}, 1 \times \mathbf{B}, 4 \times \mathbf{C}), 3$ for $2(1 \times \mathbf{B}$, $2 \times \mathbf{C}), 4$ for $3(1 \times \mathbf{A}, 1 \times \mathbf{B}, 2 \times \mathbf{C}), 4$ for $4(1 \times \mathbf{A}, 1 \times \mathbf{B}, 2 \times \mathbf{C})$, and 7 for $\mathbf{5}(2 \times \mathbf{A}, 1 \times \mathbf{B}, 4 \times \mathbf{C})$, and (2) both the neutral and ionic valence bond structures were used: this resulted in 12 structures for $1(2 \times \mathbf{A}, 1 \times \mathbf{B}, 4 \times \mathbf{C}, 1 \times \mathbf{D}, 4 \times \mathbf{E}), 6$ for $2(1 \times \mathbf{B}$, $2 \times \mathbf{C}, 1 \times \mathbf{D}, 2 \times \mathbf{E}), 7$ for $3(1 \times \mathbf{A}, 1 \times \mathbf{B}, 2 \times \mathbf{C}, 1 \times \mathbf{D}, 2 \times \mathbf{E})$, 7 for $4(1 \times \mathbf{A}, 1 \times \mathbf{B}, 2 \times \mathbf{C}, 1 \times \mathbf{D}, 2 \times \mathbf{E})$, and 12 for $5(2 \times \mathbf{A}$, $1 \times \mathbf{B}, 4 \times \mathbf{C}, 1 \times \mathbf{D}, 4 \times \mathbf{E})$. Note that for the linear molecules 1 and $\mathbf{5}$, the number of structures $\mathbf{A}, \mathbf{C}$, and $\mathbf{E}$ is doubled, due to degeneracies of $x$ and $y$. The Gallup and Norbeck scheme ${ }^{61}$ is used to calculate the weights of the individual, non-orthogonal, VB structures (see also Table S9 (ESI $\dagger$ ) for the Chirgwin and Coulson $^{62}$ weights). The advantage of these weights is that they are always positive, and sum to 1 .

\section{Results and discussion}

\section{Energy decomposition analysis}

For comparison with existing literature, we also performed energy decomposition analyses for the molecules 1-5 (Table 1 and Table S8, ESI $\dagger$ ). For 1, the linear structure was used in these calculations. Two different fragment sets were used: (1) in which the central carbon atom is prepared in its ${ }^{5} \mathrm{~S}$ state, and triplet states for the ligands (referred to as open-shell), and (2) in which the carbon atom is prepared in a ${ }^{1} \mathrm{D}$ state with singlet states for the ligands (referred to as closed-shell). In Table 1, the orbital interaction terms for the different fragments are listed for 1-5. According to Frenking and co-workers, ${ }^{3}$ the bonding situation is best described for fragments with the smallest orbital interactions. Note that in this study, we have chosen for three fragments in contrast to the earlier work, ${ }^{3}$ as we also have three fragments in our VB study. The reference molecule 4, allene, has the smallest orbital interaction energy $\left(\Delta E_{\text {orb}}\right)$ for the open-shell fragments, while the orbital

Table 1 The orbital interaction energy $\left(\mathrm{kcal} \mathrm{mol}^{-1}\right)$ as provided by the energy decomposition analysis; fragments are either open shell, or closed shell

\begin{tabular}{lllc}
\hline Compound & $\begin{array}{l}\Delta E_{\text {orb }} \text { open } \\
\text { shell }\end{array}$ & $\begin{array}{l}\Delta E_{\text {orb }} \text { closed } \\
\text { shell }\end{array}$ & $\Delta E_{\text {orb }}(\mathrm{OS})-\Delta E_{\text {orb }}(\mathrm{CS})$ \\
\hline $\mathbf{1}$ & -609.91 & -603.80 & -6.11 \\
$\mathbf{2}$ & -508.84 & -537.26 & 28.42 \\
$\mathbf{3}$ & -531.65 & -566.94 & 35.29 \\
$\mathbf{5}$ & -514.27 & -697.34 & 183.07 \\
& -609.71 & -108.70 & -501.01
\end{tabular}

interaction energy is considerably larger for the closed-shell fragments. This result further confirms that $\mathbf{4}$ can be considered as having two electron sharing bonds. The gold(I) complex 5, that is typically described as having dative bonds, ${ }^{41-43}$ shows the smallest orbital interaction for the closed-shell fragments. It has a significantly higher $\Delta E_{\text {orb }}$ for the open-shell fragments. Again, this result is in line with chemical intuition and corroborates the assignment of two dative bonds from the CO ligands to a central atom, here gold.

Now, we turn to the carbone compounds 1-3. The obtained differences between the open-shell and closed-shell $\Delta E_{\text {orb }} \mathrm{S}$ in these cases is considerably smaller. This suggests that the assignment of covalent or dative bonding is not straightforward in these cases. The open-shell fragment case is favored for molecules 2 and 3, whereas the closed-shell fragment case is favored for 1. This might lead to the temptation to interpret these results in a way that would assign $\mathbf{1}$ as a carbone, while 2 and 3 could be best described as having covalent, electron sharing, bonds. However, the differences between the open and closed-shell fragment $\Delta E_{\text {orb }}$ is rather small (around $30 \mathrm{kcal} \mathrm{mol}^{-1}$ ), so these EDA results leave some ambiguity, and it can be expected that $\mathbf{2}$ and $\mathbf{3}$ can have (considerable) carbone character.

\section{Valence bond self consistent field calculations}

To analyze the bonding situation in the molecules 1-5 in a direct manner, we have performed valence bond self-consistent field (VBSCF) calculations using the structures depicted in Scheme 2. The Gallup and Norbeck (GN) weights for the two sets of calculations (set one with only the neutral VB structures and set two that also includes the ionic structures) are listed in Table 2.

We consider the allene 4 first. The VBSCF calculation with only the neutral structures shows a high weight for the covalent structure (A), a negligible weight for the carbone structure (B) and some importance for structure $\mathbf{C}$, which has a lone-pair on the central carbon (similar to a carbene), and one electron

Table 2 The combined GN weights of the different types of valence bond structures in the two sets of calculations: first, the weights are listed when only the neutral structures are considered, second, the weights of the structures when the ionic structures are included as well (indicated with an asterisk)

\begin{tabular}{llllll}
\hline Compound & $W_{\text {covalent }}(\mathrm{A})$ & $W_{\text {carbone }}(\mathrm{B})$ & $W_{\text {carbene }}(\mathrm{C})$ & $W_{\text {ionic }}(\mathrm{D})$ & $W_{\text {ionic }}(\mathrm{E})$ \\
\hline $\mathbf{1}$ & 0.601 & 0.013 & 0.385 & & \\
$\mathbf{1}^{*}$ & 0.747 & 0.026 & 0.085 & 0.001 & 0.141 \\
$\mathbf{2}$ & & & & & \\
$\mathbf{2}^{*}$ & - & 0.157 & 0.843 & & \\
& & 0.096 & 0.064 & 0.242 & 0.598 \\
$\mathbf{3}$ & 0.720 & 0.022 & 0.258 & & \\
$\mathbf{3}^{*}$ & 0.779 & 0.029 & 0.078 & 0.015 & 0.099 \\
$\mathbf{4}$ & 0.894 & 0.003 & 0.103 & & \\
$\mathbf{4}^{*}$ & 0.960 & 0.002 & 0.031 & 0.000 & 0.007 \\
$\mathbf{5}$ & 0.006 & 0.753 & 0.241 & & \\
$\mathbf{5}^{*}$ & 0.004 & 0.440 & 0.056 & 0.441 & 0.058
\end{tabular}


sharing bond with one of the ligands, and a dative bond with the other. Upon inclusion of the ionic components to the bonding, the importance of the covalent structure A increases, whereas the combined weight of VB structure $\mathbf{C}$ decreases; the weight of the carbone structure $\mathbf{B}$ is not affected and the weights of the ionic structures are also insignificant. In line with the EDA analysis, 4 can be considered as having two covalent, double bonds.

The situation for reference molecule $\mathbf{5}$ is different: when only the neutral VB structures are taken into consideration, the dominant structure is the VB structure with dative bonds from $\mathrm{CO}$ to gold. The carbene-like structure $\mathbf{C}$ also has a considerable contribution to the wavefunction. Upon including the ionic structures, this picture changes drastically: the weight of structure B decreases, and it gets as important as ionic structure D. The weight of structures $\mathbf{C}$ declines considerably, and its weight becomes equal to that of the ionic structures $\mathbf{E}$. The weight of the pure covalent structure $\mathbf{A}$ is negligible in both sets of calculations. Hence, the OC-Au bond in $\mathbf{5}$ can be described as a hybrid between dative bonding $\mathbf{B}$ and the ionic structure $\mathbf{D}$ that possesses two covalent bonds as well. This picture is also in line with the EDA, although the information of the contributing ionic structure $\mathbf{D}$ is missing from the EDA.

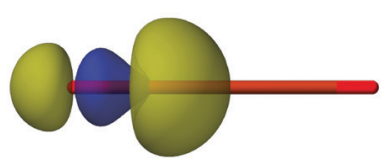

$\sigma-\mathrm{CO}$

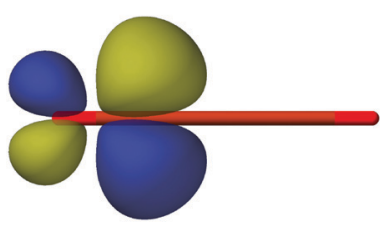

$\pi-\mathrm{CO}$
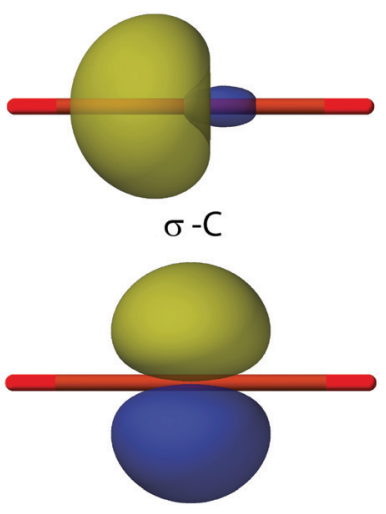

$\pi-\mathrm{C}$
Fig. 1 The symmetry unique optimized VBSCF orbitals for 1.
After the discussion of the reference compounds $\mathbf{4}$ and 5, where it was seen that the bonding in $\mathbf{4}$ can be assigned in a clear-cut way as being an electron sharing bond, and the bonding in $\mathbf{5}$ as dative $\mathbf{B}$ with ionic/covalent contributions $\mathbf{D}$, we can turn our attention to the carbones $\mathbf{1}$ and 3, as they behave rather similarly. For both compounds, the covalent structure $\mathbf{A}$ is dominant, and it gains in weight upon including the ionic structures. Unexpectedly, the weight of the carbone structure $\mathbf{B}$ is rather low, and it is clear from the VBSCF point of view that both 1 and 3 cannot be described solely by a Lewis structure that has two dative bonds. When the ionic structures are not included in the calculation, the structures $\mathbf{C}$ with one dative bond are important for the description of the molecules. However, as soon as the ionic structures are added to the wavefunction, the weights of structures $\mathbf{C}$ decreases, and the ionic structures $\mathbf{E}$ get a non-negligible weight. Thus, $\mathbf{1}$ and $\mathbf{3}$ can be described as covalent structures with important contributions from the ionic structures and mixed dative/electron sharing structures $\mathbf{C}$. The latter have one dative bond to the central carbon atom each. The VBSCF orbitals of 1 (Fig. 1) do not show any abnormalities and can be considered as regular $\sigma$ and $\pi$ orbitals localized on the CO fragment and the central $\mathrm{C}$ atom.

\section{Geometry dependence of the Valence Bond wavefunctions}

One can wonder whether the VBSCF results obtained for $\mathbf{1}$ are biased by the chosen linear geometry of $\mathbf{1}$, while the equilibrium geometry of $\mathbf{1}$ is bent (for the discussion of bond angles in carbones and related compounds, see ref. 3, 13 and 63-68). Therefore, we performed a scan of the $\angle \mathrm{C}-\mathrm{C}-\mathrm{C}$ angle at the CCSD/cc-pVTZ level of theory (keeping $\angle \mathrm{C}-\mathrm{C}-\mathrm{C}$ frozen and optimized the rest of the molecule) and did VBSCF calculations on the obtained geometries. The energy and the weights as a function of $\angle \mathrm{C}-\mathrm{C}-\mathrm{C}$ are depicted in Fig. 2. The results show that the VBSCF and the CCSD calculations agree that $\mathbf{1}$ is not linear, and that the linear structure is a transition state connecting to the two bent minima. However, the barrier calculated using VBSCF theory is considerably higher than the CCSD one. One might consider the bond angle to be a)

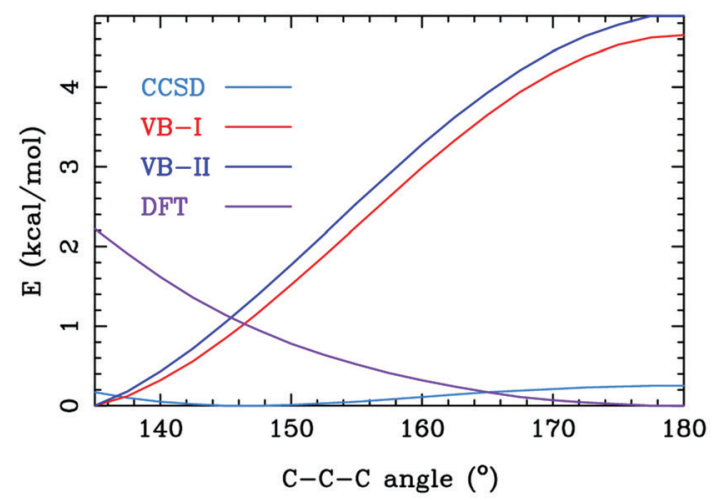

b)

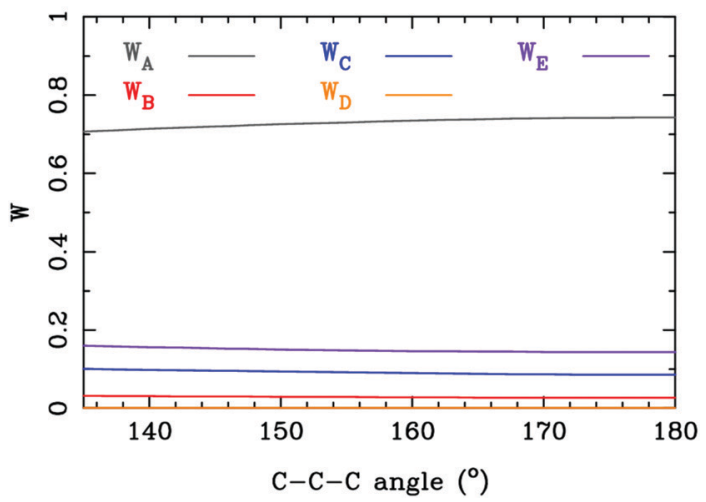

Fig. 2 (a) The CCSD, VB-I (only neutral structures), VB-II (neutral and ionic structures) and DFT (BP86/TZ2P) energies (kcal mol ${ }^{-1}$ ), relative to the lowest point, as a function of the $\mathrm{C}-\mathrm{C}-\mathrm{C}$ angle $\left({ }^{\circ}\right)$; (b) the weights of the VB structures as a function of the $\mathrm{C}-\mathrm{C}-\mathrm{C}$ angle $\left(^{\circ}\right.$ ). 
Table 3 Optimized geometries of 1 with selected valence bond structures: listed are the total energies (au), relative energies with respect to the energy of entry $1\left(\mathrm{kcal} \mathrm{mol}^{-1}\right)$, the $\mathrm{C}-\mathrm{C}-\mathrm{C}$ angles $\left(^{\circ}\right)$ and the $\mathrm{C}-\mathrm{C}$ distances (A). For entries 6 and 7 , the geometry was constrained to be linear. As a reference, in entry 8 , the CCSD/cc-pVTZ optimized geometry is listed

\begin{tabular}{llllll}
\hline Entry & $E_{\text {VBSCF }}$ & $E_{\text {rel }}$ & Structures & $\angle$ C-C-C & $R_{\mathrm{C}-\mathrm{C}}$ \\
\hline 1 & -263.324650 & 0.00 & $\mathbf{A}+\mathbf{B}+\mathbf{C}+\mathbf{D}+\mathbf{E}$ & 133.0 & 1.324 \\
2 & -263.296732 & 17.52 & $\mathbf{A}+\mathbf{B}+\mathbf{C}$ & 133.5 & 1.313 \\
3 & -263.158396 & 104.33 & $\mathbf{A}$ & 124.6 & 1.307 \\
4 & -263.097006 & 142.85 & $\mathbf{B}$ & 48.9 & 6.152 \\
5 & -263.182792 & 89.02 & $\mathbf{C}$ & 124.1 & 1.316 \\
6 & -263.144968 & 112.75 & $\mathbf{A}$ & 180.0 & 1.289 \\
7 & -262.744954 & 363.77 & $\mathbf{B}$ & 180.0 & 1.334 \\
8 & -264.256849 & & CCSD & 146.3 & 1.285 \\
\hline
\end{tabular}

quasi-linear when using CCSD. ${ }^{3}$ Also, the degree of bending is substantially different: VBSCF theory predicts a considerably smaller $\angle \mathrm{C}-\mathrm{C}-\mathrm{C}$ than CCSD. The inclusion of ionic structures in the VBSCF calculations has only a small effect on the height of the transition state and the $\mathrm{C}-\mathrm{C}-\mathrm{C}$ angle. The composition of the wavefunction, however, remains constant on the reaction path. Structure A remains the dominant structure on the whole path, and structure $\mathbf{B}$, the carbone structure remains to contribute rather little. This means that VB results obtained for the linear geometry are also valid for the bent structure of $\mathbf{1}$ and that the geometry has hardly any influence on the composition of the wavefunction.

Full geometry optimizations are also possible with VBSCF theory. ${ }^{52,69,70}$ When all structures are included a bent geometry is obtained (Table 3, entry 1 ). The obtained OC-C bond length is a bit elongated with respect to the reference CCSD structure, and the angle is more bent. Omission of the ionic structures in the VBSCF wavefunction does not lead to any significant changes in the geometry of $\mathbf{1}$ (entry 2). Surprisingly, if only the covalent structure $\mathbf{A}$ is included in the VBSCF wavefunction (entry 3), a more bent structure is found, instead of the expected linear geometry. The carbone structure $\mathbf{B}$ alone does not lead to bonded minimum (entry 4), and dissociation of the CO moieties is observed. The dative bonding is not strong enough to keep the molecule bonded. A resonance hybrid of structures $\mathbf{C}$ leads to a similarly bent structure as has been obtained in the optimization of structure A only (entry 5).

When a linear geometry is enforced, and structure $\mathbf{A}$ is only used in the wavefunction (entry 6), the energy obtained is higher than the bent structure A geometry, as expected. If only carbone structure $\mathbf{B}$ is taken (entry 7), while enforcing linearity, a bonded situation is found. However, the energy is considerably higher than the dissociated products.

A last issue to address is whether the OC-C bond has some degree of charge-shift bond character ${ }^{71,72}$ dative bonding in metal-ligand complexes has a high degree of charge-shift bond character, ${ }^{72}$ hence, the dative part of the OC-C bond in $\mathbf{1}$ may also have a high degree of charge-shift bond character. Therefore, we elongated one of the OC-C bonds and calculated the VBSCF energy, including the ionic structures. The covalent structure $\mathbf{A}$ is the structure with the lowest energy (Fig. 3) in the region of the equilibrium bond length. On the dissociation

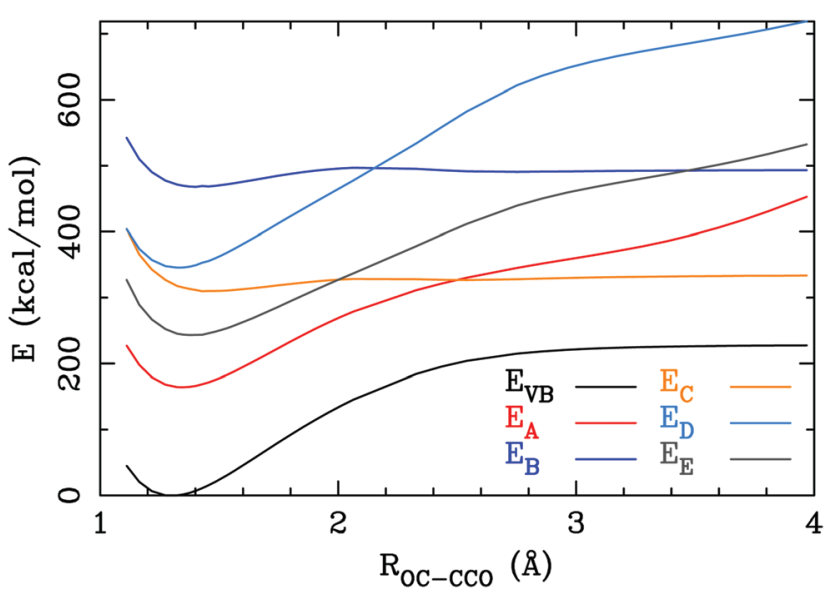

Fig. 3 The total $\mathrm{VBSCF}$ energy $\left(E_{\mathrm{VB}}\right)$, and the energy of the covalent structures $\left(E_{\mathrm{A}}\right)$, the carbone structure $\left(E_{\mathrm{B}}\right)$, the carbene structures $\left(E_{\mathrm{C}}\right)$ and the ionic structures $\left(E_{\mathrm{D}}\right)$ and $\left(E_{\mathrm{E}}\right)$ as a function of the $\mathrm{OC}-\mathrm{CCO}$ distance.

path, this structure has a minimum that nearly coincides with the minimum in the total energy curve. Ionic structures $\mathbf{E}$ are next higher in energy: this curve also possesses a minimum near the equilibrium structure. Although interactions between the structures considerably lowers the energy, the dissociation curves do not resemble the curves that are obtained for typical charge-shift bonds like the one in $\mathrm{F}_{2},{ }^{73}$ hence, the OC-C bond cannot be assigned to the charge-shift bond family.

\section{Conclusions}

Here we have reported a valence bond study of typical molecules that were assigned to possess dative bonding towards a carbon atom, and therefore termed as carbones. We have shown that, in comparison with archetypical molecules classified with electron sharing $\left(\mathrm{H}_{2} \mathrm{C}=\mathrm{C}=\mathrm{CH}_{2}\right)$ and dative bonds $\left([\mathrm{OC}-\mathrm{Au}-\mathrm{CO}]^{+}\right)$, the carbone VB structure is not important in the description of the bond. The covalent structure prevails with relatively high contributions of ionic structures. The importance of the mixed dative/electron sharing bond structures shows that the bond in the so called carbone molecules do have dative bond character, but these bonds cannot be assigned to purely dative. The carbone structure itself proves to be unstable, and does not lead to a bonded molecule. The OC-C bond also does not show any significant sign of charge-shift bond character.

Based on these results, we see that indeed the OC-C bond in 1 and 3 are distinct from "normal" electron sharing bonds and from dative bonding. As bonding in molecules covers a large spectrum, it might be best classified to be placed in between these two extremes.

\section{Conflicts of interest}

There are no conflicts to declare. 


\section{Acknowledgements}

This research used resources (Summit and SummitDev) of the Oak Ridge Leadership Computing Facility at the Oak Ridge National Laboratory, which is supported by the Office of Science of the U.S. Department of Energy under Contract No. DE-AC0500OR22725 (DD and ESP). This work was sponsored by NWO Exact and Natural Sciences for the use of supercomputer facilities (contract no. 17197 7095). We would like to thank the Center for Information Technology of the University of Groningen for their support and for providing access to the Peregrine high performance computing cluster. J. E. M. N. K. acknowledges funding from the Netherlands Organisation for Scientific Research (NWO START-UP grant (740.018.014)).

\section{References}

1 R. Tonner, F. Öxler, B. Neumüller, W. Petz and G. Frenking, Carbodiphosphoranes: The chemistry of divalent carbon(0), Angew. Chem., Int. Ed., 2006, 45, 8038-8042.

2 R. Tonner and G. Frenking, $\mathrm{C}(\mathrm{NHC})_{2}$ : Divalent Carbon $(0)$ Compounds with N-Heterocyclic Carbene Ligands-Theoretical Evidence for a Class of Molecules with Promising Chemical Properties, Angew. Chem., Int. Ed., 2007, 46, 8695-8698.

3 R. Tonner and G. Frenking, Divalent Carbon(0) Chemistry, Part 1: Parent Compounds, Chem. - Eur. J., 2008, 14, 3260-3272.

4 R. Tonner, G. Heydenrych and G. Frenking, First and second proton affinities of carbon bases, ChemPhysChem, 2008, 9, 1474-1481.

5 S. Klein, R. Tonner and G. Frenking, Carbodicarbenes and Related Divalent Carbon(0) Compounds, Chem. - Eur. J., 2010, 16, 10160-10170.

$6 \mathrm{~W}$. Petz and G. Frenking, Carbodiphosphoranes and Related Ligands, in Transition Metal Complexes of Neutral $\eta^{1}$-Carbon Ligands, ed. R. Chauvin and Y. Canac, 2010, vol. 30, pp. 49-92.

7 G. Frenking and R. Tonner, Divalent carbon(0) compounds, Pure Appl. Chem., 2009, 81, 597-614.

8 A. Clark and H. M. Seip, The potential function for the CCC bending in carbon suboxide, Chem. Phys. Lett., 1970, 6, 452-456.

9 R. L. Livingston and C. N. Ramachandra Rao, The molecular structure of carbon suboxide, J. Am. Chem. Soc., 1959, 81, 285-287.

$10 \mathrm{H}$. Mackle and L. E. Sutton, The structure of carbon suboxide by the method of electron diffraction, Trans. Faraday Soc., 1951, 47, 937-942.

11 P. Jensen and J. W. C. Johns, The Infrared Spectrum of Carbon Suboxide in the $\nu_{6}$ Fundamental Region: Experimental Observation and Semirigid Bender Analysis, J. Mol. Spectrosc., 1986, 118, 248-266.

12 A. Ellern, T. Drews and K. Seppelt, The Structure of Carbon Suboxide, $\mathrm{C}_{3} \mathrm{O}_{2}$, in the Solid State, Z. Anorg. Allg. Chem., 2001, 627, 73-76.
$13 \mathrm{~J}$. Koput, An ab initio study on the equilibrium structure and CCC bending energy levels of carbon suboxide, Chem. Phys. Lett., 2000, 320, 237-244.

14 F. M. Bickelhaupt and E. J. Baerends, Kohn-Sham Density Functional Theory: Predicting and Understanding Chemistry, in Reviews in Computational Chemistry, ed. K. B. Lipkowitz and D. B. Boyd, Wiley-VCH, New York, 2000, vol. 15, pp. 1-86.

15 G. Frenking, R. Tonner, S. Klein, N. Takagi, T. Shimizu, A. Krapp, K. K. Pandey and P. Parameswaran, New bonding modes of carbon and heavier group 14 atoms $\mathrm{Si}-\mathrm{Pb}$, Chem. Soc. Rev., 2014, 43, 5106-5139.

16 C. Esterhuysen and G. Frenking, Distinguishing Carbones from Allenes by Complexation to AuCl, Chem. - Eur. J., 2011, 17, 9944-9956.

17 T. H. Wang, W. C. Chen and T. G. Ong, Carbodicarbenes or Bent Allenes, J. Chin. Chem. Soc., 2017, 64, 124-132.

18 G. Frenking and M. Hermann, Gilbert Lewis and the Model of Dative Bonding, in Chemical Bond I: 100 Years Old and Getting Stronger, ed. D. M. P. Mingos, 2016, vol. 169, pp. 131-156.

19 M. Alcarazo, C. W. Lehmann, A. Anoop, W. Thiel and A. Fürstner, Coordination chemistry at carbon, Nat. Chem., 2009, 1, 295-301.

20 G. Frenking, Dative Bonds in Main-Group Compounds: A Case for More Arrows!, Angew. Chem., Int. Ed., 2014, 53, 6040-6046.

21 D. Himmel, I. Krossing and A. Schnepf, Dative Bonds in Main-Group Compounds: A Case for Fewer Arrows!, Angew. Chem., Int. Ed., 2014, 53, 370-374.

$22 \mathrm{H}$. Schmidbaur, Réplique: A New Concept for Bonding in Carbodiphosphoranes?, Angew. Chem., Int. Ed., 2007, 46, 2984-2985.

23 S. Shaik and P. C. Hiberty, A Chemist's Guide to Valence Bond Theory, John Wiley \& Sons, Inc., Hoboken, New Jersey, 2008.

24 J. Turek, B. Braïda and F. De Proft, Bonding in Heavier Group 14 Zero-Valent Complexes-A Combined Maximum Probability Domain and Valence Bond Theory Approach, Chem. - Eur. J., 2017, 23, 14604-14613.

$25 \mathrm{~J}$. H. van Lenthe and G. G. Balint-Kurti, The valence-bond scf (VB SCF) method: Synopsis of theory and test calculation of OH potential energy curve, Chem. Phys. Lett., 1980, 76, 138-142.

26 J. H. van Lenthe and G. G. Balint-Kurti, The valence-bond self-consistent field method (VB-SCF): Theory and test calculations, J. Chem. Phys., 1983, 78, 5699-5713.

27 B. J. Duke and R. W. A. Havenith, Implications of the complete basis set limit in valence bond theory: a case study of molecular hydrogen, Theor. Chem. Acc., 2016, $135,82$.

28 P. C. Hiberty, Reconciling simplicity and accuracy: compact valence bond wave functions with breathing orbitals, $J$. Mol. Struct., 1997, 398-399, 34-43.

29 P. C. Hiberty, J. P. Flament and E. Noizet, Compact and accurate valence bond functions with different orbitals for different configurations: application to the two-configuration description of $\mathrm{F}_{2}$, Chem. Phys. Lett., 1992, 189, 259-265. 
30 P. C. Hiberty, S. Humbel, C. P. Byrman and J. H. van Lenthe, Compact valence bond functions with breathing orbitals: Application to the bond dissociation energies of $\mathrm{F}_{2}$ and $\mathrm{FH}$, J. Chem. Phys., 1994, 101, 5969-5976.

31 P. C. Hiberty and S. Shaik, Breathing-orbital valence bond method - a modern valence bond method that includes dynamic correlation, Theor. Chem. Acc., 2002, 108, 255-272.

32 M. Zielinski, R. W. A. Havenith, L. W. Jenneskens and J. H. van Lenthe, A comparison of approaches to estimate the resonance energy, Theor. Chem. Acc., 2010, 127, 19-25.

33 Z. Rashid, J. H. van Lenthe and R. W. A. Havenith, Resonance and Aromaticity: An Ab Initio Valence Bond Approach, J. Phys. Chem. A, 2012, 116, 4778-4788.

34 J. Gerratt, General Theory of Spin-Coupled Wave Functions for Atoms and Molecules, in Advances in Atomic and Molecular Physics, ed. D. Bates and I. Esterman, Academic Press, 1971, vol. 7, pp. 141-221.

35 J. Gerratt and M. Raimondi, The spin-coupled valence bond theory of molecular electronic structure. I. Basic theory and application to the ${ }^{2} \Sigma^{+}$states of $\mathrm{BeH}$, Proc. R. Soc. London, Ser. A, 1980, 371, 525-552.

36 D. L. Cooper, J. Gerratt and M. Raimondi, Studies of molecular states using spin-coupled valence-bond theory, Faraday Symp. Chem. Soc., 1984, 19, 149-163.

37 D. L. Cooper; J. Gerratt and M. Raimondi, Modern Valence Bond Theory, in Advances in Chemical Physics: Ab initio Methods in Quantum Chemistry - II, ed. K. P. Lawley, John Wiley \& Sons, London, 1987, vol. LXIX, pp. 319-397.

38 W. J. Hunt, P. J. Hay and W. A. Goddard III, Self-Consistent Procedures for Generalized Valence Bond Wavefunctions. Applications $\mathrm{H}_{3}, \mathrm{BH}, \mathrm{H}_{2} \mathrm{O}, \mathrm{C}_{2} \mathrm{H}_{6}$, and $\mathrm{O}_{2}$, J. Chem. Phys., 1972, 57, 738-748.

39 P. J. Hay, W. J. Hunt and W. A. Goddard III, Generalized valence bond description of simple alkanes, ethylene, and acetylene, J. Am. Chem. Soc., 1972, 94, 8293-8301.

40 W. A. Goddard III, T. H. Dunning Jr., W. J. Hunt and P. J. Hay, Generalized valence bond description of bonding in low-lying states of molecules, Acc. Chem. Res., 1973, 6, 368-376.

41 H. V. R. Dias, C. Dash, M. Yousufuddin, M. A. Celik and G. Frenking, Cation Gold Carbonyl Complex on a Phosphine Support, Inorg. Chem., 2011, 50, 4253-4255.

42 M. A. Celik, C. Dash, V. A. K. Adiraju, A. Das, M. Yousufuddin, G. Frenking and H. V. R. Dias, End-On and Side-On $\pi$-Acid Ligand Adducts of Gold(I): Carbonyl, Cyanide, Isocyanide, and Cyclooctyne Gold(I) Complexes Supported by N-Heterocyclic Carbenes and Phosphines, Inorg. Chem., 2013, 52, 729-742.

43 L. Nunes dos Santos Comprido, J. E. M. N. Klein, G. Knizia, J. Kästner and A. S. K. Hashmi, Gold(I) Vinylidene Complexes as Reactive Intermediates and Their Tendency to $\pi$ Backbond, Chem. - Eur. J., 2016, 22, 2892-2895.

44 E. J. Baerends, ADF2017.01, SCM, Theoretical Chemistry, Vrije Universiteit, Amsterdam, The Netherlands, http:// www.scm.com, 2017.
45 C. Fonseca Guerra, J. G. Snijders, G. te Velde and E. J. Baerends, Towards an order-N DFT method, Theor. Chem. Acc., 1998, 99, 391-403.

46 G. te Velde, F. M. Bickelhaupt, E. J. Baerends, C. Fonseca Guerra, S. J. A. van Gisbergen, J. G. Snijders and T. Ziegler, Chemistry with ADF, J. Comput. Chem., 2001, 22, 931-967.

47 E. van Lenthe, E. J. Baerends and J. G. Snijders, Relativistic regular two-component Hamiltonians, J. Chem. Phys., 1993, 99, 4597-4610.

48 E. van Lenthe, E. J. Baerends and J. G. Snijders, Relativistic total energy using regular approximations, J. Chem. Phys., 1994, 101, 9783-9792.

49 E. van Lenthe, R. van Leeuwen, E. J. Baerends and J. G. Snijders, Relativistic regular two-component Hamiltonians, Int. J. Quantum Chem., 1996, 57, 281-293.

50 M. J. Frisch, G. W. Trucks, H. B. Schlegel, G. E. Scuseria, M. A. Robb, J. R. Cheeseman, G. Scalmani, V. Barone, G. A. Petersson, H. Nakatsuji, X. Li, M. Caricato, A. V. Marenich, J. Bloino, B. G. Janesko, R. Gomperts, B. Mennucci, H. P. Hratchian, J. V. Ortiz, A. F. Izmaylov, J. L. Sonnenberg, D. Williams-Young, F. Ding, F. Lipparini, F. Egidi, J. Goings, B. Peng, A. Petrone, T. Henderson, D. Ranasinghe, V. G. Zakrzewski, J. Gao, N. Rega, G. Zheng, W. Liang, M. Hada, M. Ehara, K. Toyota, R. Fukuda, J. Hasegawa, M. Ishida, T. Nakajima, Y. Honda, O. Kitao, H. Nakai, T. Vreven, K. Throssell, J. A. Montgomery Jr., J. E. Peralta, F. Ogliaro, M. J. Bearpark, J. J. Heyd, E. N. Brothers, K. N. Kudin, V. N. Staroverov, T. A. Keith, R. Kobayashi, J. Normand, K. Raghavachari, A. P. Rendell, J. C. Burant, S. S. Iyengar, J. Tomasi, M. Cossi, J. M. Millam, M. Klene, C. Adamo, R. Cammi, J. W. Ochterski, R. L. Martin, K. Morokuma, O. Farkas, J. B. Foresman and D. J. Fox, Gaussian 16 Rev. C.01, Wallingford, CT, 2016.

51 D. E. Woon and T. H. Dunning, Gaussian basis sets for use in correlated molecular calculations. III. The atoms aluminum through argon, J. Chem. Phys., 1993, 98, 1358-1371.

52 J. H. van Lenthe, F. Dijkstra and R. W. A. Havenith, TURTLE - A gradient VBSCF Program. Theory and Studies of Aromaticity. In Valence Bond Theory, ed. D. L. Cooper, Elsevier, Amsterdam, 2002, vol. 10, pp. 79-112.

53 J. Verbeek, J. H. Langenberg, C. P. Byrman, F. Dijkstra, R. W. A. Havenith, J. J. Engelberts, M. Zielinski, Z. Rashid and J. H. van Lenthe, TURTLE, an ab initio $V B / V B S C F$ program, Utrecht, The Netherlands, 1988-2016.

54 M. F. Guest, I. J. Bush, H. J. J. van Dam, P. Sherwood, J. M. H. Thomas, J. H. van Lenthe, R. W. A. Havenith and J. Kendrick, The GAMESS-UK electronic structure package: algorithms, developments and applications, Mol. Phys., 2005, 103, 719-747.

55 T. H. Dunning, Gaussian basis sets for use in correlated molecular calculations. I. The atoms boron through neon and hydrogen, J. Chem. Phys., 1989, 90, 1007-1023.

56 D. Figgen, G. Rauhut, M. Dolg and H. Stoll, Energyconsistent pseudopotentials for group 11 and 12 atoms: adjustment to multi-configuration Dirac-Hartree-Fock data, J. Chem. Phys., 2005, 311, 227-244. 
57 K. A. Peterson and C. Puzzarini, Systematically convergent basis sets for transition metals. II. Pseudopotential-based correlation consistent basis sets for the group $11(\mathrm{Cu}, \mathrm{Ag}$, $\mathrm{Au}$ ) and 12 (Zn, Cd, Hg) elements, Theor. Chem. Acc., 2005, 114, 283-296.

58 D. Feller, The role of databases in support of computational chemistry calculations, J. Comput. Chem., 1996, 17, 1571-1586.

59 B. P. Pritchard, D. Altarawy, B. Didier, T. D. Gibsom and T. L. Windus, A New Basis Set Exchange: An Open, Up-todate Resource for the Molecular Sciences Community, J. Chem. Inf. Model., 2019, 59, 4814-4820.

60 K. L. Schuchardt, B. T. Didier, T. Elsethagen, L. Sun, V. Gurumoorthi, J. Chase, J. Li and T. L. Windus, Basis Set Exchange: A Community Database for Computational Sciences, J. Chem. Inf. Model., 2007, 47, 1045-1052.

61 G. A. Gallup and J. M. Norbeck, Population analyses of valence-bond wavefunctions and $\mathrm{BeH}_{2}$, Chem. Phys. Lett., 1973, 21, 495-500.

62 B. H. Chirgwin and C. A. Coulson, The electronic structure of conjugated molecules. VI, Proc. R. Soc. London, Ser. A, 1950, 201, 196-209.

63 S. Böttger, M. Gruber, J. Eike Münzer, G. M. Bernard, N.-J. H. Kneusels, C. Poggel, M. Klein, F. Hampel, B. Neumüller, J. Sundermeyer, V. K. Michaelis, R. Tonner, R. R. Tykwinski and I. Kuzu, Solvent-Induced Bond-Bending Isomerism in Hexaphenyl Carbodiphosphorane: Decisive Dispersion Interactions in the Solid State, Inorg. Chem., 2020, 59, 12054-12064.

64 F. Dahcheh, D. Martin, D. W. Stephan and G. Bertrand, Synthesis and Reactivity of a CAAC-Aminoborylene Adduct:
A Hetero-Allene or an Organoboron Isoelectronic with Singlet Carbenes, Angew. Chem., Int. Ed., 2014, 53, 13159-13163. 65 H. Braunschweig, I. Krummenacher, M.-A. Légaré, A. Matler, K. Radacki and Q. Ye, Main-Group Metallomimetics: Transition Metal-like Photolytic CO Substitution at Boron, J. Am. Chem. Soc., 2017, 139, 1802-1805.

66 P. J. Quinlivan and G. Parkin, Flexibility of the Carbodiphosphorane, $\left(\mathrm{Ph}_{3} \mathrm{P}\right)_{2} \mathrm{C}$ : Structural Characterization of a Linear Form, Inorg. Chem., 2017, 56, 5493-5497.

67 R. Appel, U. Baumeister and F. Knoch, Darstellung und Molekülstruktur aminosubstituierter Carbodiphosphorane, Chem. Ber., 1983, 116, 2275-2284.

68 W.-L. Li, H.-T. Liu, T. Jian, G. V. Lopez, Z. A. Piazza, D.-L. Huang, T.-T. Chen, J. Su, P. Yang, X. Chen, L.-S. Wang and J. Li, Bond-bending isomerism of $\mathrm{Au}_{2} \mathrm{I}_{3}{ }^{-}$: competition between covalent bonding and aurophilicity, Chem. Sci., 2016, 7, 475-481.

69 F. Dijkstra and J. H. van Lenthe, Gradients in Valence Bond theory, Chem. Phys. Lett., 1999, 310, 553-556.

70 F. Dijkstra and J. H. van Lenthe, Gradients in Valence Bond theory, J. Chem. Phys., 2000, 113, 2100-2108.

71 G. Sini, P. Maitre and P. C. Hiberty, Covalent, ionic and resonating single bonds, J. Mol. Struct., 1991, 229, 163-188.

72 S. Shaik, D. Danovich, J. Morrison Galbraith, B. Braïda, W. Wu and P. C. Hiberty, Charge-shift bonding: A new and unique form of bonding, Angew. Chem., Int. Ed., 2020, 59, 984-1001.

73 H. Zhang, D. Danovich, W. Wu, B. Braïda, P. C. Hiberty and S. Shaik, Charge-Shift Bonding Emerges as a Distinct Electron-Pair Bonding Family from Both Valence Bond and Molecular Orbital Theories, J. Chem. Theory Comput., 2014, 10, 2410-2418. 